\title{
Seamless Mixed Reality Tracking in Tabletop Reservoir Engineering Interaction
}

\author{
Paul Lapides, Nicole Sultanum, Ehud Sharlin, Mario Costa Sousa \\ Department of Computer Science, University of Calgary, \\ 2500 University Drive NW, Calgary, T2N 1N4, AB, Canada \\ paul.lapides@gmail.com, \{nbsultan, ehud, smcosta\}@ucalgary.ca
}

\begin{abstract}
In this paper we present a novel mixed reality tracking system for collaborative tabletop applications that uses decorative markers and embedded application markers to create a continuous and seamless tracking space for mobile devices. Users can view and interact with mixed reality datasets on their mobile device, such as a tablet or smartphone, from distances both far and very near to the tabletop. We implement the tracking system in the context of a collaborative reservoir engineering tool that brings together many experts who need a private workspace to interact with unique datasets, which is supported by our system.
\end{abstract}

\section{Categories and Subject Descriptors}

H.5.1 [Multimedia information Systems]: Artificial, augmented and virtual realities. J.2 [Physical Sciences and Engineering]: Earth and atmospheric sciences, Engineering.

\section{General Terms}

Design, Human Factors.

\section{Keywords}

Tabletop interaction, mixed-reality, reservoir engineering.

\section{INTRODUCTION}

Tabletop interaction is motivated by the collaborative nature afforded by its form factor. Users can gather around the table, gain access to its content by their mere physical presence, and control the interaction flow by touching the tabletop surface. While the collaborative nature of the shared tabletop is empowering, it arguably also conflicts with and negates the natural need for privacy and personal space in interaction, which is often a crucial element in many collaborative activities [10]. Our goal is to create an environment that will allow users to fully benefit from the collaborative aspects of the tabletop, while also being able to access and interact with a personal layer of information that is seamlessly integrated with the tabletop interface.

We present a mixed reality tabletop interface for reservoir engineering exploration. The interface integrates direct tabletop interaction with a 3D mixed reality layer which is supported by ubiquitous markers. Our interface (Figure 1) allows users to either interact directly with the tabletop content, via touch, or interact Permission to make digital or hard copies of all or part of this work for personal or classroom use is granted without fee provided that copies are not made or distributed for profit or commercial advantage and that copies bear this notice and the full citation on the first page. To copy otherwise, to republish, to post on servers or to redistribute to lists, requires prior specific permission and/or a fee.

AVI '12, May 21-25, 2012, Capri Island, Italy.

Copyright (C) 2012 ACM 978-1-4503-1287-5/12/05... \$10.00. with a mixed reality layer that is superimposed over the tabletop, using a set of embedded markers for tracking.

Our interface is designed in the context of interactive visualization techniques for reservoir engineering exploration, and is part of our ongoing efforts to research and design solutions for this field [13]. Reservoir engineering is a unique and challenging domain for user experience design because there is a strong need for collaboration between experts in different fields that may have diverse perspectives and sometimes conflicting solution strategies [12].

Our approach is unique in that it integrates ubiquitous markers into the reservoir engineering tabletop interface environment. Our design is based on a set of far-field markers that decorate the tabletop frame and enable mixed reality tracking when users are further away from the tabletop, as well as a set of semi-covert near-field markers which can be added to any interactive entity in the interface and enable tracking when users are examining and interacting with the tabletop content from close distances. Our design allows for continuous and seamless tracking using the two sets of tabletop markers based on the user interaction flow, distance to the tabletop, as well as changes in the interactive entities displayed on the tabletop, and occlusions.

This short paper presents our work-in-progress effort of creating an interactive tabletop environment that will allow seamless transition between the direct tabletop interactive environment and an integrated mixed reality layer which is accessed via a personal device such as a tablet or similar mobile device.

In this paper we motivate our design approach, describe our implementation and our fully functional prototype, and finally outline our future work plans. While our team does include and rely on direct contact with reservoir engineers, the interface we present in this report has not yet been formally evaluated and validated.

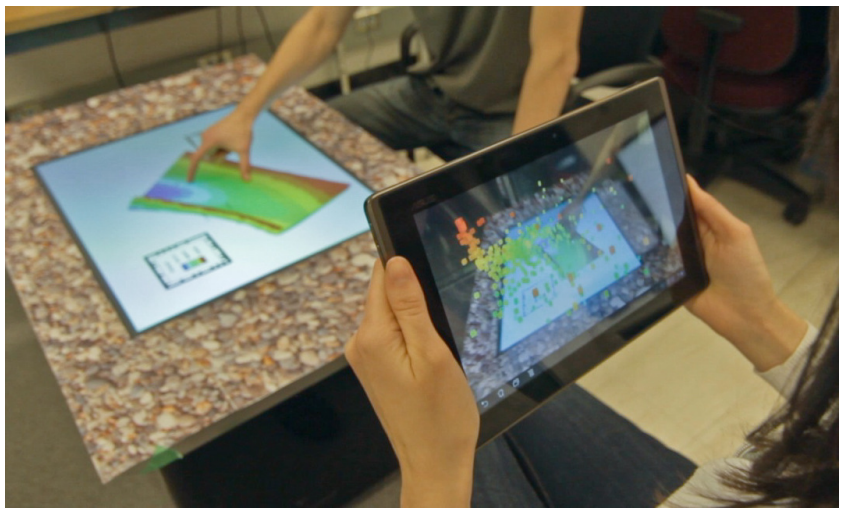

Figure 1: Two users interacting with a reservoir dataset. 


\section{RELATED WORK}

Due to its affordances to collaborative decision-making, digital tabletops have been explored in several 'serious', real-world applications, such as collaborative genome-browsing, multi-user molecule visualization, military strategy, and land use planning $[1,6,9,11]$. In the domain of geosciences and petroleum engineering, tabletops have been applied to geophysics, monitoring of oil production facilities on a management level, collaborative visualization of reservoir simulation data, and a tangible clay tabletop system for terrain modeling [4, 12, 13, 14, $15]$.

Mixed reality visualization on mobile devices has been addressed by several different tracking methods. Many techniques use a camera attached to the device to track fiduciary markers embedded on or around the objects to be tracked [5, 7]. This technique is inexpensive because many devices already have an integrated camera, and simple markers can be printed. These systems have become more robust and can more readily deal with occlusions and poor lighting conditions. However, for tracking to be maintained, the device must always be pointed at the object; the tracking will break as soon as the fiducial marker is not in the camera's view. Also, many fiducial markers have distinctive black and white patterns and must have a large size to be detected effectively. If the marker is to be shown on a screen or display, it may take a lot of valuable screen from other applications or visualizations.

A new technique was presented for tracking a device used to interact with a large display [2]. Instead of displaying large fiducials on the screen, the authors treat the screen image as a marker in and of itself. Each screen frame is analyzed and a set of contrast features is computed by a server. The image from the mobile device's camera is sent wirelessly to the server and matched to the screen image's feature set, and the device's position is calculated from this match. While this method does not rely on any explicit markers, it is currently slow and relies on a screen image with many contrast features, creating a possible limitation to the types of visualizations that can be used for tracking.

Finally, it is possible to track the mobile device with motion capture cameras or similar active tracking systems [8]. This is a very robust solution that is not impacted by the quality of the devices' camera, the direction the device is pointed, or any kind of markers. However, this method is very expensive, requiring many specialized cameras and software, as well as a dedicated space for this equipment to be set up and calibrated.

\section{TABLETOP MIXED REALITY}

The fundamental problem of mixed reality applications is to determine the position and orientation of the mobile device, or "viewing window" relative to the objects that the mixed reality augmentation is applied to. While the efforts that were mentioned previously are feasible, they are either: too slow, not stable enough, or very expensive.

\subsection{Tracking with Markers}

We attempt to tackle the problem of tracking by using the devices' onboard camera, similar to fiducials and [2]. We use the Qualcomm Augmented Reality Toolkit, which supports two different types of markers: Image Targets and Frame Markers.

Image Targets are similar to fiducials in that they are passive images that can be printed on paper or displayed on a screen.
However, unlike fiducials that are typically black and white patterns, Image Targets can be comprised of almost any color image or photograph and work on a concept similar to [2]. The photograph is analyzed off-line to produce a set of unique features, which are then searched for in the camera image during runtime. Photographs with many high contrast features distributed throughout the entire image create the best Image Targets; photos with repeating patterns or soft contrast cannot be used.

The most computationally expensive stage of the tracking is the analysis of the photograph, which is done off-line and does not negatively impact the speed of tracking, especially if many Image Targets are used in the same application. Also, the high distribution of features in an Image Target means that large parts of the marker can be occluded while still maintaining tracking (Figure 2).

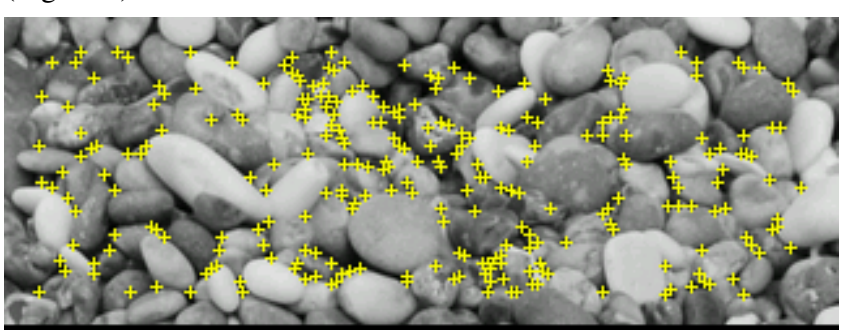

Figure 2: Ideal Image Target with high contrast features.

Frame Markers are similar to typical fiducial markers. They consist of several black and white patterns encoded around the border of the marker. What is unique about Frame Markers is that the inside of the marker can be replaced with any kind of image (Figure 4). These markers do not need to be analyzed a priori since only the border of the marker is tracked, not the image contents inside of it. Therefore, it is possible to dynamically change the contents inside of the marker when displayed on a screen. Additionally, these markers retain excellent tracking speed and react well to changing light conditions, although they doesn't support partial occlusion.

\subsection{Combining Markers}

The goal of our system is to implement fast, robust tracking for a tabletop that has minimal interference to the application while allowing users to interact with the mixed reality content on the tablet from very near to the tabletop and also further away. We combine Image Targets and Frame Markers in our tracking system to accomplish this.

\subsection{Edge Markers}

First we ensure that tablet users have tracking from far distances from the tabletop, such as from a standing position one or two feet away. This distance gives the user a macro view of the tabletop and is useful for interpreting a large collection of data quickly.

We place eight Image Targets around the edge of the tabletop: four in each corner and four along the long edges of the display (Figure 3). The configuration of edge markers can include fewer or more markers, depending on the size of the display.

In our initial prototype, the edge markers were individual photographs and did not cover the edges of the table completely. To create a more aesthetic presentation, we printed a large photograph to completely cover the tabletop border. The edge marker images themselves are cropped areas corresponding to the corners and edges of the large photo. 


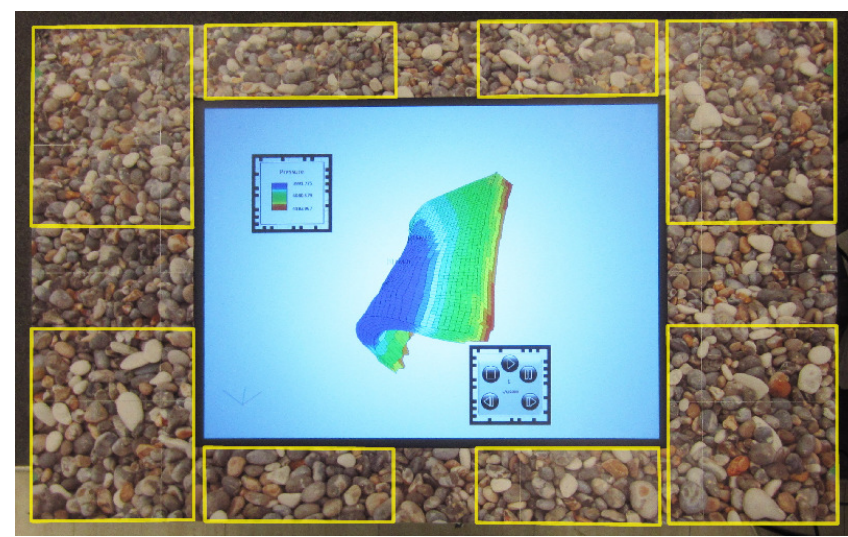

Figure 3: This photo of stones contains eight edge markers.

\subsection{Application Markers}

When the user brings the tablet close to the tabletop, the edge markers may fall out of the device's camera view causing the tracking to stop working until the user resumes pointing the device at these markers. To prevent such a disruption to the user's workflow, we display several application markers on the tabletop surface so the device's camera can see at least one marker in almost any usage case.

Frame Markers are well suited for this purpose as they are less obtrusive to the application than Image Targets, and they can be filled with application specific controls or information such as widgets, logos, and renderings. As previously mentioned, any occlusions (such as from the user's hand) will break that marker's tracking, so it is best to use more than one application marker to ensure redundancy.

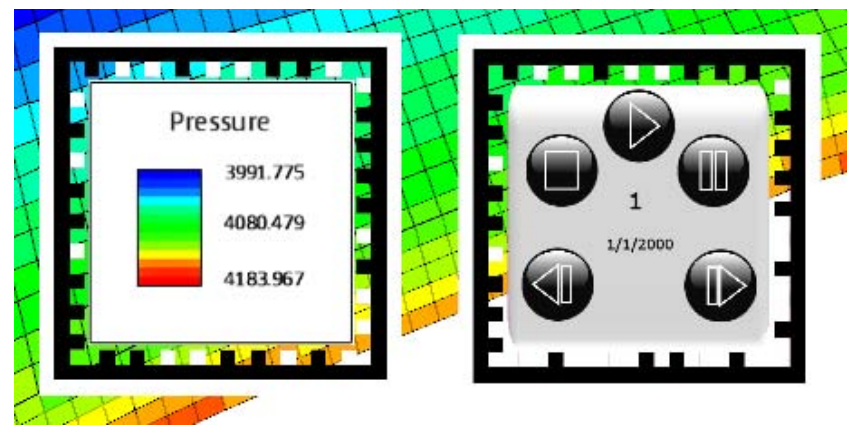

Figure 4: Two application markers, each containing a widget, can be overlaid above different visualizations.

\section{RESERVOIR ENGINEERING MIXED- REALITY}

We applied the presented concepts to an oil \& gas visualization application, building on previous work involving reservoir engineering visualization for tabletops $[12,13]$. This domain is strongly characterized for its collaborative aspect, involving highly multidisciplinary exchanges among team members [13], and thus a relevant test-bed for the concept of alternative views around a shared common element.

The centerpiece of the visualization environment is a tabletop displaying a shared reservoir model, and accessible to all users. The tablet visualization is a projection of a point-based dataset immediately over the tabletop view containing microseismic events: underground fracture events mapped to estimated spatial locations (Figure 5) [3]. The tabletop widgets, including a property color scale and a time step navigator were adapted to become application markers and provide additional tracking redundancy when the edge markers are not visible (Figure 4).

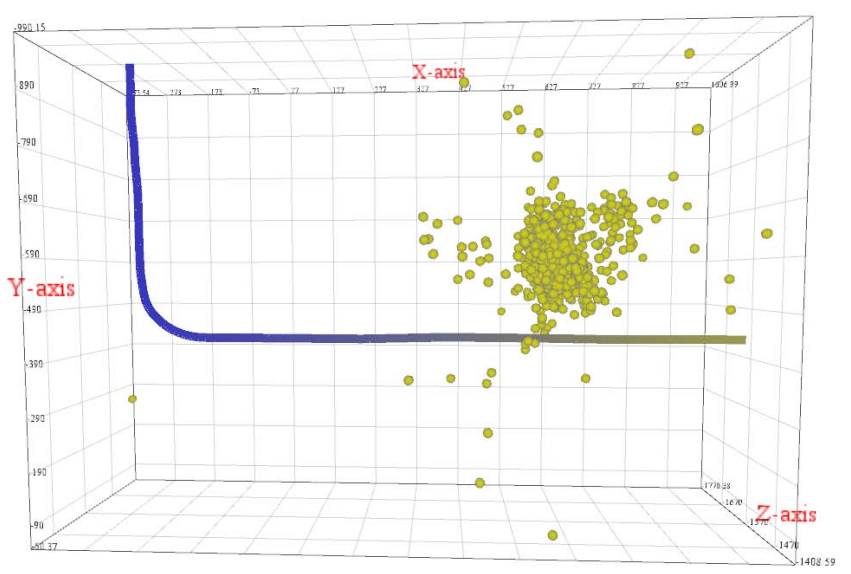

Figure 5: Microseismic data-points adjacent to a well.

The motivation for this design is that the microseismic dataset complements the understanding of the underlying model, but it may not be as relevant to the entire team as it is to the immediate expert. Following this rationale, every expert could have their own complementary datasets and additional elements set up in their personal views, with direct spatial association to the reservoir models that aggregates the data.

Our system also addresses the inherent difficulty of providing adequate spatial awareness for point-based representation. Users may now view the data from different angles, and even circle around it to acquire a better perception of the location of the micro-seismic events.

\section{LIMITATIONS AND FUTURE WORK}

Our approach has several limitations. The tracking will stop working if the user points the tablet in an upwards direction to look at the dataset from the bottom since no edge or application markers will be visible to the tablet's camera. It may be possible to mitigate this problem by placing large markers on the walls or ceiling. Another minor limitation of Frame Markers is that they must be squares, creating a small restriction to what types of widgets or data can be shown inside of them.

At our current development stage, the application markers are static entities and must remain in the same position on the screen. We will implement a wireless communication protocol between the tabletop application and the tablet so that when application markers are moved, resized, and rotated, the tablet can continue to render the mixed reality dataset from the correct perspective. Also, operations made on the tabletop application (such as rotating or translating the main reservoir model) will be mirrored in the mixed reality application so both datasets will be spatially synchronized.

Further, application markers can automatically adapt to the positions of the tablet. If the user is standing far away, there may not be any need to show application markers at all. However, if they begin to approach the tabletop, one or two application markers may begin to appear, only near that user. This approach can be used to manage application markers for several tablet users while preserving as much screen space as possible. 


\section{CONCLUSIONS}

In this paper we have presented a system that facilitates the use of personal spaces for collaborative tabletop applications. By using mobile devices as personal visualization windows, users can view information that is most relevant to them while still being able to interact with the shared data and tabletop visualization. We created a mixed reality application that uses two types of markers for tracking: edge markers that are disguised as decorative photographs lining the edges of the tabletop, and application markers that may have application specific widgets and data inside of a special border. Edge markers provide tracking at far distances while application markers handle near distance tracking, creating seamless and continuous tracking for the user regardless of where they choose to work.

We have implemented this tracking system to work with a collaborative tabletop reservoir engineering tool that overlays a point-based dataset over top of the tabletop visualization. Such an application is used collaboratively by several engineers who are experts in different areas. Each user requires unique visualizations of datasets that pertain directly to their area of expertise, while still being sensitive to the main reservoir model that is at the center of the collaboration. Our system provides users a private workspace where they can interact with their own datasets associated to the main shared view.

The awareness of the need for privacy, as well as a flexible and intuitive solution to this problem may be critical to the usability of some collaborative applications. We hope our work to create a mixed reality system that enables privacy in tabletop applications may be used as a model for other privacy sensitive applications.

\section{REFERENCES}

[1] Arciniegas, G.A., and Janssen, R. 2009. Using a touch table to support participatory land use planning. In 18th World IMACS Congress and MODSIM09 International Congress on Modelling and Simulation. Modelling and Simulation (Cairns, Australia, July 13-17, 2009).

[2] Baur, D., Boring, S., and Feiner, S. 2012. Virtual Projection: Exploring Optical Projection as a Metaphor for Multi-Device Interaction. In Proceedings of the SIGCHI Conference on Human Factors in Computing Systems (Austin, Texas, USA, May 5-10, 2012) CHI'12. ACM, New York, NY.

[3] Cipolla, C., Maxwell, S., Mack, M., Downie, R., and Schlumberger. 2011. A practical guide to interpreting microseismic measurements. In Proceedings of the North American Unconventional Gas Conference and Exhibition (The Woodlands, Texas, USA, June 14-16, 2011). Society of Petroleum Engineers.

[4] Couture, N., Rivière, G., and Reuter, P. 2008. GeoTUI: a tangible user interface for geosciences. In Proceedings of the 2nd International Conference on Tangible and Embedded Interaction (Bonn, Germany, February 18-20, 2008), TEI '08. ACM, New York, NY.

[5] Fiala, M. 2005. ARTag, a fiducial marker system using digital techniques. In IEEE Computer Society Conference on Computer Vision and Pattern Recognition (San Diego, CA, USA, June 20-26, 2005). v.2, p.590-596, June 2005.
[6] Forlines, C., and Lilien, R. 2008. Adapting a single-user, single-display molecular visualization application for use in a multi-user, multi-display environment. In Proceedings of the working conference on Advanced Visual Interfaces (Napoli, Italy, May 28-30, 2008).

[7] Piekarski, W., Avery, B., Thomas, B., and Malbezin, P. 2003. Hybrid Indoor and Outdoor Tracking for Mobile 3D Mixed Reality. In 2nd International Symposium on Mixed and Augmented Reality (Tokyo, Japan, October 2003).

[8] Sandor, C., Kuroki, T., Uchiyama, S., and Yamamoto, H. 2007. Exploring Visuo-Haptic Mixed Reality.

[9] Scott, S., Allavena, A., Cerar, K., Franck, G., Hazen, M., Shuter, T., and Colliver, C. 2010. Investigating tabletop interfaces to support collaborative decision-making in maritime operations. In Proceedings of ICCRTS 2010: International Command and Control Research and Technology Symposium (Santa Monica, CA, USA, June 2224, 2010).

[10] Scott, S., Grant, K., and Mandryk, R. 2003. System guidelines for co-located, collaborative work on a tabletop display. In Proceedings of the $8^{\text {th }}$ European Conference on Computer Supported Cooperative Work (Helsinki, Finland, September 14-18, 2003) ECSCW'03. Kluwer Academic Publishers, Norwell.

[11] Shaer, O., Kol, G., Strait, M., Fan, C., Grevet, C., and Elfenbein, S. 2010. G-Nome Surfer: a tabletop interface for collaborative exploration of genomic data. In Proceedings of the 28th international conference on Human factors in computing systems (Atlanta, GA, USA, April 10-15, 2010) CHI '10. ACM, New York, NY.

[12] Sultanum, N., Somanath, S., Sharlin, E. and Costa Sousa, M. 2011. "Point it, split it, peel it, view it": techniques for interactive reservoir visualization on tabletops. In Proceedings of the ACM International Conference on Interactive Tabletops and Surfaces (Kobe, Japan, November 13-16, 2011) ITS '11. ACM, New York, NY.

[13] Sultanum, N., Sharlin, E., Costa Sousa, M., Miranda-Filho, D., and Eastick, R. 2010. Touching the depths: Introducing tabletop interaction to reservoir engineering. In Proceedings of ACM International Conference on Interactive Tabletops and Surfaces (Saarbrücken, Germany, November 7-10, 2010) ITS '10. ACM, New York, NY.

[14] Tateosian, L., Mitasova, H., Harmon, B., Fogleman, B., Weaver, K., and Harmon, R. 2010. TanGeoMS: Tangible Geospatial Modeling System. IEEE Transactions on Visualization and Computer Graphics, v.16 n.6, p.16051612, November 2010.

[15] The Information Store and Infusion Development. Petrotrek for the microsoft surface. www.istore.com.

[16] University of Calgary DSpace Entry, 2012. Video: Seamless Mixed Reality Tracking in Tabletop Reservoir Engineering Interaction. 